\title{
Preparation and Characterization of Polyurethane Nanocomposites Using Vietnamese Montmorillonite Modified by Polyol Surfactants
}

\author{
C. N. Ha Thuc, ${ }^{1,2}$ H. T. Cao, ${ }^{3}$ D. M. Nguyen, ${ }^{1}$ M. A. Tran, ${ }^{1}$ Laurent Duclaux, \\ A.-C. Grillet, ${ }^{4}$ and H. Ha Thuc ${ }^{5}$ \\ ${ }^{1}$ Department of Polymer and Composite Materials, Faculty of Materials Science, University of Science, \\ National University of HCM City (VNU-HCM), Ho Chi Minh City 70000, Vietnam \\ ${ }^{2}$ Laboratoire de Chimie Moléculaire et Environnement, Polytech'Annecy-Chambéry, Université de Savoie, \\ 73370 Le Bourget du Lac Cedex, France \\ ${ }^{3}$ Department of Magnetic and Biomedical Materials, Faculty of Materials Science, University of Science, \\ National University of HCM City (VNU-HCM), Ho Chi Minh City 70000, Vietnam \\ ${ }^{4}$ Laboratoire de LOCIE, Polytech' Annecy-Chambéry, Université de Savoie, 73370 Le Bourget du Lac Cedex, France \\ ${ }^{5}$ Department of Polymer, Faculty of Chemistry, University of Science, National University of HCM City (VNU-HCM), \\ Ho Chi Minh City 70000, Vietnam
}

Correspondence should be addressed to C. N. Ha Thuc; htcnhan@hcmus.edu.vn

Received 6 September 2013; Revised 11 December 2013; Accepted 11 December 2013; Published 16 March 2014

Academic Editor: Sheng-Rui Jian

Copyright (C) 2014 C. N. Ha Thuc et al. This is an open access article distributed under the Creative Commons Attribution License, which permits unrestricted use, distribution, and reproduction in any medium, provided the original work is properly cited.

This study focuses on the preparation of thermoplastic polyurethane (TPU) nanocomposite using Vietnamese montmorillonite (MMT) as the reinforced phase. The MMT was previously modified by intercalating polyethylene oxide (PEO) and polyvinyl alcohol (PVA) molecules between the clay layers. X-ray diffraction (XRD) results of organoclays revealed that galleries of MMT were increased to $18.2 \AA$ and $27 \AA$ after their intercalation with PEO and PVA, respectively. Thermoplastic polyurethane (TPU) nanocomposites composed of $1,3,5$, and $7 \%$ wt organoclays were synthesized. The result of XRD and transmission electron microscopic (TEM) analyses implied that the PEO modified MMT was well dispersed, at 3\%wt, in polyurethane matrix. Fourier Transform Infrared Spectroscopic (FTIR) has confirmed this result by showing the hydrogenous interaction between the urethane linkage and $\mathrm{OH}$ group on the surface of silicate layer. Thermogravimetric (TG) showed that the organoclay samples also presented improved thermal stabilities. In addition, the effects of the organoclays on mechanical performance and water absorption of the PU nanocomposite were also investigated.

\section{Introduction}

Polyurethane (PU) is a versatile polymeric material with desirable properties, for example, high abrasion resistance, tear strength, excellent shock absorption, flexibility, and elasticity. By blending with inorganic fillers its performance has been improved further. Since 1998, PU/clay nanocomposites $[1,2]$ were developed, but the good dispersion of clay, being a key parameter in the synthesis of clay/polymer nanocomposites, depends on the clay mineral purity and its modification.
As an example, the purity of a natural montmorillonite (MMT) can be improved by removing the associated gangue minerals such as sand, gravel, quartz, feldspars, calcite, iron oxides, and humic acids which are oftenly co-existed with MMT in natural clay [3-6]. The purity of the MMT has a strong effect on engineering properties of polymer nanocomposites especially on elongation and impact resistance [7]. Thus the highest purity possible of this kind of filler is required for nanocomposite elaboration.

The aim of clay modification is to render the layered silicates miscible with polymer matrices like polyurethane, by 
lowering the surface energy, improving the wetting characteristics of the polymer matrix, and enlarging the interlayer spacing of clay. The normally hydrophilic silicate surface should be converted to an organophilic one, allowing the intercalation of many engineering polymers. After Xiong et al. [8], there are two main factors that should be considered in forming polymer/clay nanocomposites; firstly, the organic modifier molecules must enter in interlayer galleries in enlarging the interlayer space of MMT, and then result the easier interaction of polymer molecules or monomers and clay layers. Generally, the modification is processed by ionexchange reactions with cationic surfactants including primary, secondary, tertiary, and quaternary alkylammonium or alkylphosphonium cations [7, 9-11] or by intercalation in clay of hydrophilic polymers, such as polyethylene oxide (PEO), or polyvinyl alcohol (PVA) [10, 12-16].

Many researchers studied the PU nanocomposites to improve the mechanical performance of polyurethane and reduce the production cost $[7,10,11]$. However, such approach has some disadvantages, such as the reduction of ductility, and the decrease of water resistance. There are very few reports on TPU composites and nanocomposites prepared by bulk and melt processing $[17,18]$. Recently, much effort has been conducted to find new methods for preparing PU/modified MMT. Wang and Pinnavaia [1] or Zilg et al. [19] reported an intercalated PU/MMT by in situ polymerization of Toluene diisocyanate (TDI) and a mixture of polyol and MMT. Cheng et al. [20] proposed an intercalation method in solution, as PU prepolymer was inserted into the MMT intergallery in solution conditions followed by a curing procedure with 1,4-butanediol. To further improve the properties of PU/clay nanocomposites, alternate functional modifiers which can react with polymer are being used to prepare polymer/clay nanocomposites. For example, in the study of Chen et al. [21], the authors found exfoliation of clay layers in PU nanocomposites, when clay was treated by aminolauric acid (12COOH) and benzidine (BZD) separately, as shown by X-Ray diffraction patterns and TEM. The mechanical property of polyurethane nanocomposite with benzidine modified clay showed much better than that of 12-aminolauric acid treated clay, and it was explained by the difference in the interaction between the swelling agent and polyurethane molecular chain. 12-Aminolauric acid contained only one terminal $-\mathrm{NH}_{2}$ group, which might react with a minute amount of unreacted - NCO to form urea, while both of the two terminal $-\mathrm{NH}_{2}$ groups in benzidine could participate in the reaction. Therefore, cross-linking structure can be formed by benzidine and linear chains by 12-aminolauric. In another study of Tien and Wei [22], the effect of exfoliation behavior on mechanical properties in tethered/untethered PU nanocomposites was investigated by using 1,2 , and 3 hydroxyl groups in the molecules of organic treatment of layered silicates, which acted as pseudochain extenders for polyurethane prepolymer. X-Ray analysis of polyurethane nanocomposites prepared by solution method confirmed that with the increase of numbers of hydroxyl groups in quaternary ammonium ions, the dispersion of layered silicates in polyurethanes transformed from an intercalated to an exfoliated state. Later, Pattanayak and Jana
$[18,23,24]$ have studied the in situ reaction of polyurethane nanocomposites with reactive Cloisite $30 \mathrm{~B}$ through bulk polymerization. Clay-polymer tethering reaction occurred under conditions of high shear stress by using a conventional batch mixer. Two processing methods were used in this synthesis of nanocomposite materials. In the first method, the chain extender, 1,4-butanediol, was added after the addition of clay; in the second method, the chain extension reaction was carried out before the addition of clay. Unlike the solution polymerization method conducted by Tien and Wei [22], the addition of clay before chain extension reaction led to poor dispersion of clay particles. With the confirmation of FTIR and rheological data, the clay exfoliation was found to be influenced mainly by strong interaction between modified clay and PU chain.

Based on the report of these authors about the claytethered polyurethane nanocomposites $[18,21,22,25]$ in the present study, we describe the preparation PU/clay nanocomposites from purified Vietnamese montmorillonite which is modified, via solution method, by polyethylene oxide (PEO) and polyvinyl alcohol (PVA) molecules. The modifier agents having the hydroxyl functional groups are considered to react with PU chains as pseudochain extenders for polyurethane prepolymer. Thermoplastic polyurethane nanocomposite composed of $1,3,5$, and 7\%wt of organoclays were then prepared via in situ polymerization from crystalline polypropylene glycol (PPG) polyols, and 4,4'-diphenylmethane diisocyanate (1,4-MDI), using 1,4-butanediol $(1,4-\mathrm{BD})$ as chain extender. This paper describes also the morphology and some basic properties of the prepared PU nanocomposite.

\section{Experimental}

2.1. Materials and Chemicals. Vietnamese montmorillonite (MMT) minerals (LamDong $\mathrm{Mg}^{2+}$-montmorillonite) was obtained by purification of bentonite from the Clay Minerals Depository Hiep Phu at the LamDong province (south of Vietnam) using the purification process reported in our previous study [26]. The polymers used in this work for the modification of clay were polyethylene oxide PEO $\left(\mathrm{M}_{n}=10000\right)$ and polyvinyl alcohol PVA $\left(\mathrm{M}_{n}=8700\right)$ from Aldrich Chemical Company, Inc. For the synthesis of PU, 4,4'-diphenylmethane diisocyanate (1,4-MDI, Aldrich), 1,4-butanediol (1,4-BD, Aldrich), and polypropylene glycol (PPG, $\mathrm{M}_{n}=1200$, Aldrich) were used as received from Aldrich Chemical Company. The PPG, 1,4-BD, and 1,4-MDI reagents were vacuum dried at $80^{\circ} \mathrm{C}$ for $24 \mathrm{~h}$ prior to use.

2.2. Preparation of $P E O$ and $P V A$ Modified MMT. The clay used was the purified MMT obtained from our previous purification procedure [26]. In the literature, two processes are reported for the PEO or PVA intercalation of clay [12, $13,15]$ solution intercalation and melt intercalation. In this work, the solution intercalation was performed because it allows intercalating polymers with little or no polarity into layered structure and facilitates the production of orientedclay intercalated layer $[7,27]$.

In a typical experiment, the selected polymer and silicate were weighed according to the designated ratio. A weighed 
amount of purified clay (1 $\mathrm{g}$ ) was dispersed in a known volume of polymer solution at $60^{\circ} \mathrm{C}$, for 4 hours. The solid sample was collected by centrifugation and then washed two times with distilled water before keep it in a vacuum oven $\left(\sim 60^{\circ} \mathrm{C}\right)$. The initial polymer concentrations in aqueous solution $\left(\mathrm{C}_{0}\right)$ were in the range $0.01,0.03$, and $0.05 \mathrm{M}$ for $\mathrm{PEO}$ and $0.03,0.05$, and $0.1 \mathrm{M}$ for PVA.

The success of intercalation of PEO-MMT and PVAMMT samples was checked by X-Ray diffraction (XRD), and the saturation ratio of polymer intercalated in samples should be determined by differential scanning calorimetry (DSC).

2.3. Synthesis of PU/Clay Nanocomposites. Polyurethane nanocomposites containing 1,3,5, and 7\% content (weight percent) of modified MMTs were prepared from PEO and PVA modified MMTs (PEO-MMT obtained PEO/MMT = 0.3/1 ratio and PVA-MMT obtained PVA/MMT $=1$ ratio). For the preparation, $40 \mathrm{~g}$ of dry PPG were added to a stoichiometric ratio of dried modified MMT (MO-MMT) powder in a $250 \mathrm{~mL}$ flask with a mechanical stirrer at ambient temperature. Additional mixing was performed via an ultrasonicator $(100 \mathrm{~W}$, nominal frequency of $35 \mathrm{kHz})$ at ambient temperature and then the mixture was heated to $90^{\circ} \mathrm{C}$ for $2 \mathrm{~h}$ to remove residual water. Then $1,4-\mathrm{MDI}$ was added in a molar ratio of $2: 1$ (relatively to PPG) to the mixture of PPG and modified MMT at $80^{\circ} \mathrm{C}$ to obtain the prepolyurethane/modified MMT material after $1.5 \mathrm{~h}$ of reaction. After the NCO-terminated prepolymer was formed, $3 \mathrm{~g}$ of 1,4-BD were added to the prepolyurethane/MO-MMT under vigorous stirring for $60 \mathrm{~s}$ at $80^{\circ} \mathrm{C}$. Dibutyltin dilaurate $(0.02 \mathrm{wt} \%)$ was added to the reacting solution reaction at $80^{\circ} \mathrm{C}$ and subsequently, the prepolyurethane/MO-MMT was immediately poured in a metal mold with dimensions of $18.5 \times 15.5 \times 2\left(\mathrm{~cm}^{3}\right)$ and then cured for $2 \mathrm{~h}$ at $80^{\circ} \mathrm{C}$ in a vacuum oven to form a polyurethane (PU)/modified MMT nanocomposite film after demoulding.

\subsection{Characterization Techniques}

2.4.1. X-Ray Diffraction (XRD). The modified MMT were characterized by X-Ray Powder Diffraction (WAXRD). Analyses were performed at the $\mathrm{CuK}_{\alpha 1}$ radiation by using a BraggBrentano $(\theta, 2 \theta)$ mode goniometer (CGR, $\theta$ 60) equipped with an X-Ray generator (INEL XRG 3000) set at $35 \mathrm{kV}$ tension and $30 \mathrm{~mA}$ current, a point proportional detector (4545 LND), and a curve quartz monochromator (curve radius $=250 \mathrm{~mm}$ ). The scanning rate was $0.02^{\circ} / \mathrm{s}$ over a range of $2 \theta=2-20^{\circ}$ for one-dimensional diffraction. XRD pattern were collected from $2^{\circ}$ to $50^{\circ}(2 \theta)$ and performed on oriented sample prepared by the deposit of suspension of clays on glass slide.

For the nanocomposite characterization, small-angle X-Ray diffraction (SAXRD) experiments were performed directly on the film samples which have thickness of $0.5 \mathrm{~mm}$. The measurements were carried out with a Bruker D8 advance diffractometer (graphite monochromator, using $\mathrm{Cu}-\mathrm{K}_{\alpha 1}$ radiation, step scan of $0.02^{\circ}$, and $1 \mathrm{~s}$ per step) in the $0.5-102 \theta$ range. The XRD line profile analysis was performed with TOPAS P software (Bruker AXS, Karlsruhe, Germany) using a split pseudovoigt profile function to determine the $2 \theta$ position of the 001 reflection. The refined profiles were used for the determination of reflection positions.

2.4.2. Transmission Electronic Microscopy (TEM). The samples for the transmission electron microscopy (TEM, JEOL 3010) study were microtomed using a cryogenic ultramicrotome system (Leica Ultracut Uct) into $50 \mathrm{~nm}$ thick slices. Subsequently, $3 \mathrm{~nm}$ thick amorphous carbon layer was deposited on to these slices, supported on 200-mesh copper nets for TEM observation.

2.4.3. Thermogravimetric Analyses (TGA). The thermal degradations of the polymers were observed by thermogravimetric analysis (TGA), at a heating rate of $10^{\circ} \mathrm{C} / \mathrm{min}$ in the temperature range $30-12000^{\circ} \mathrm{C}$. The atmosphere used was air and the sample weights were $80-100 \mathrm{mg}$.

2.4.4. Differential Scanning Calorimetry (DSC). The thermal transitions of polymers were observed by differential scanning calorimetry (DSC 2020 of TA instrument), at a heating rate of $10^{\circ} \mathrm{C} / \mathrm{min}$ under nitrogen purge of $30 \mathrm{~mL} / \mathrm{min}$. The sample sizes were $3-10 \mathrm{mg}$ in a sealed aluminum pan.

2.4.5. Dynamic Mechanical Analysis Properties (DMA). The main features determined in this study are the module $\mathrm{E}^{\prime}$ and loss factor $(\tan \delta)$ of materials. DMA was carried out in a tensile mode using a frequency of $5 \mathrm{~Hz}$ on Rheometric Scientific RAC 815 de Metravib RDS. All tests were carried out at room temperature.

2.4.6. Water Adsorption Measurement. Water absorption measurement were carried out in a desiccator containing a high humidity rate ( $98 \%$ water) controlled by $\mathrm{CuSO}_{4}$ solution and the sample mass uptake was determined as a function of time.

\section{Results and Discussion}

3.1. Characterization of the PEO Modified MMT (PEO-MMT). Figure 1 shows the XRD patterns of the montmorillonite modified by $\mathrm{PEO}$ using solution intercalation at different PEO content. The melt and solution intercalation of the PEO/MMT system with different ratio has been discussed carefully in the studies of Shen et al. $[12,13]$. In our study, the $d_{001}$ basal spacings of the obtained hybrid materials increase together with increasing PEO content. Figure 1 shows a $d_{001}$ basal distance $\sim 14.5 \AA\left(2 \theta \sim 6^{\circ}\right)$, for $\mathrm{PEO} / \mathrm{MMT}=0.1$ hybrid material with compared to $d_{001} \sim 10 \AA$ for a dry MMT. This distance increases up to $d_{001} \sim 18.2 \AA$ for a fully intercalated $\mathrm{MMT}$ (at PEO/MMT $=0.3$ and 0.5 ).

According to the literature [12-14], PEO has the chain thickness of about $4 \AA$ arranged in the interlayer forming the monolayer or bilayer with the zig-zag or spiral conformation. The report is adequate to this study, our XRD results show that the intercalation of PEO monolayer is obtained for the $\mathrm{PEO} / \mathrm{MMT}$ ratios equal to 0.1 corresponding to the $d_{001}$ 


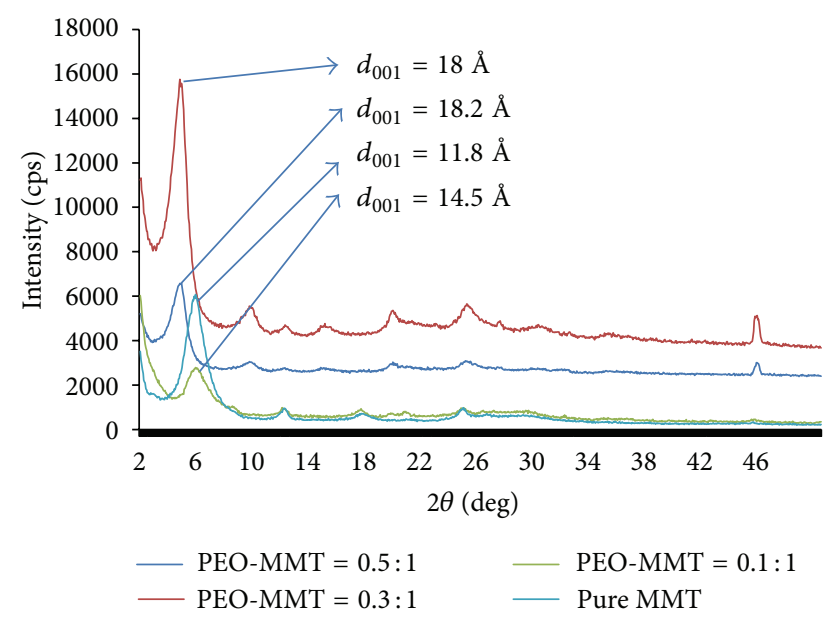

FIgURE 1: X-Ray patterns of PEO-MMT mixtures as a function of modifier/host ratios $(\mathrm{PEO} / \mathrm{MMT}=0.1,0.3$, and 0.5$)$.

distance $\sim 13.80 \AA$ and that at the ratios equal to 0.3 and 0.5 , the clay interlayer was expanded to about $17-18 \AA$, implying that a PEO bilayer insertion has occurred. In addition, except $d_{001}$, the hk. bands $\left(2 \theta=2-20^{\circ}\right)$ of MMT such as $d_{002}$ $\left(\sim 10^{\circ}\right), d_{003}\left(\sim 15.1^{\circ}\right)$, and $d_{004}\left(\sim 20^{\circ}\right)$ still showed up in the spectra indicating the stability of the layer structure after the intercalation of PEO. And otherwise, the two reflections observed at about $12.5^{\circ}$ and $25^{\circ}$ are characteristic for kaolinite impurity which is not intercalated by PEO.

DSC characterization of the PEO-MMT complex brought out the changing of $\mathrm{PEO}$ crystalline peaks after reaction with MMT. This could be explained by the transformation of the crystalline PEO into low crystallinity phase inserted into the MMT galleries. Moreover, the interaction between the adsorbed PEO and the polar groups on the surface layers of montmorillonite has prevented the PEO crystallization.

The melting endothermic peak of PEO was nearly unobserved in DSC thermograms (Figure 2) of the $\mathrm{PEO} / \mathrm{MMT}=$ 0.1 modified MMT sample, which did not also show crystalline PEO diffraction lines at $2 \theta \sim 21^{\circ}$ in its XRD spectra. This result may be due to the PEO chains being confined in between the layer silicates, cannot crystallize. In the $\mathrm{PEO} /$ $\mathrm{MMT}=0.3$ and 0.5 modified MMTs samples, the endothermic peak shown at $40^{\circ} \mathrm{C}$ and $63^{\circ} \mathrm{C}$, respectively, relating to the melting of the PEO crystallites, was observed. Crystalline PEO can be formed as well as the excess amount of PEO which cannot be intercalated into the clay galleries (from the ratio of 0.3 ). The melting temperature of sample is decreased, in comparison with pure PEO melting at about $67^{\circ} \mathrm{C}$, when fewer of the PEO content is used for the modification of MMT. This has been ascribed to disruption of large scale crystallite formation by the presence of the large amount of clay.

Therefore, the XRD and DSC results suggest that the $\mathrm{PEO}-\mathrm{MMT}=0.3$ ratio almost corresponds to a saturation $\mathrm{PEO}$ ratio value of intercalation in MMT. Thus this PEO concentration was chosen for the synthesis of further PU nanocomposites.

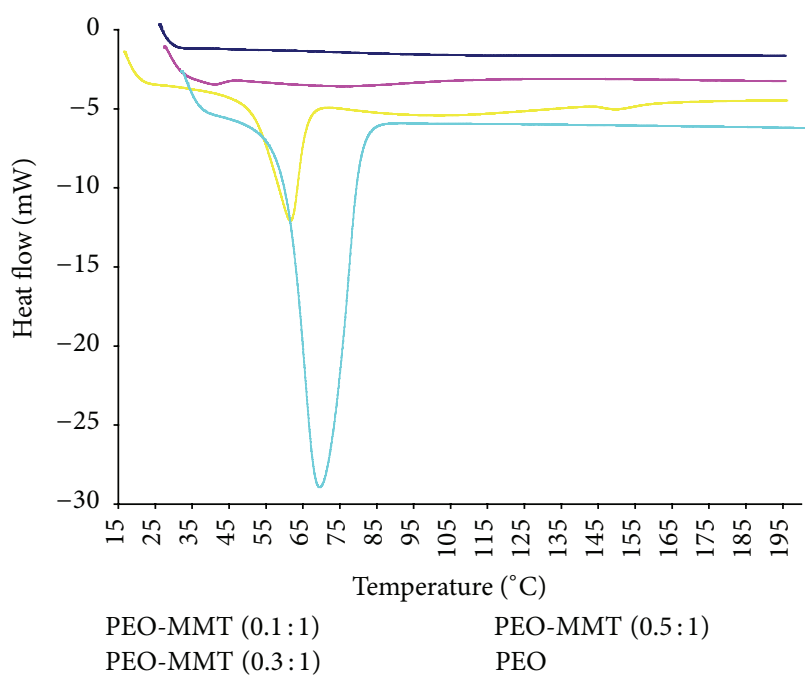

FIGURE 2: DSC analysis of PEO-MMT mixtures realized by intercalation in solution method.

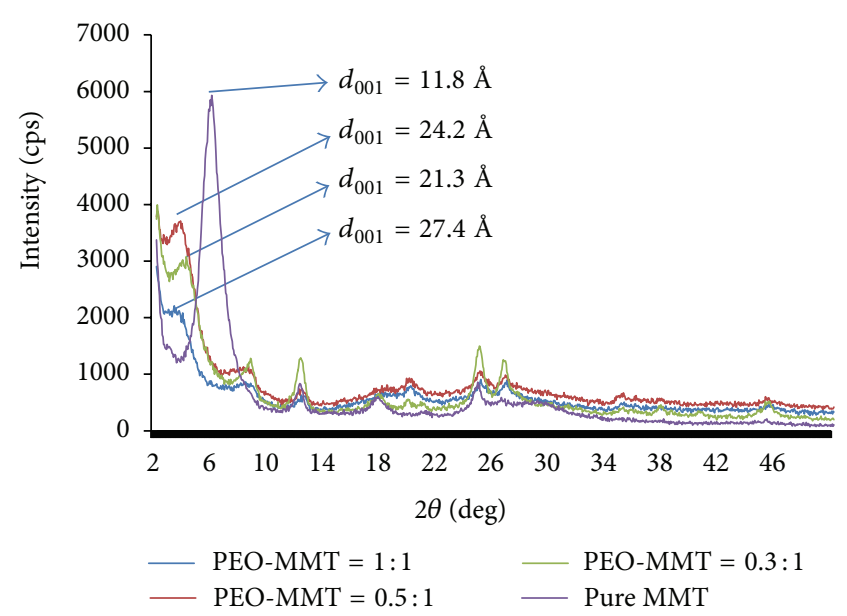

FIGURE 3: XRD patterns of PVA-MMT mixtures in function of modifier ratios/host weight ratios $(\mathrm{PVA} / \mathrm{MMT}=0.3,0.5$ and 1$)$.

\subsection{Characterization of the PVA Modified MMT (PVA-MMT} System). The modification of MMT layers was achieved by reaction with polyvinyl alcohol (PVA). Comparison of the intercalated gallery height with that of the pristine MMT $(9.7 \AA)$ is shown in XRD scans, in Figure 3, for 0.3, 0.5, and $1 \mathrm{PVA} / \mathrm{MMT}$ ratios. The basal spacing increase systematically for the increasing of PVA loading, from $0.3\left(d_{001}=21 \AA\right)$ to 1 $\left(d_{001}=27 \AA\right)$.

This result was also reported by Strawhecker and Manias [16] which suggests that the existence of periodic assemblies of intercalated MMT layers can lead to large clay d-spacing proportional to PVA concentration in the hybrid material. So in our case, while the $50 \%$ wt of PVA (ratio of $1 / 1$ ) gave the best result in enhancing the layer distance, this PVA concentration was chosen for the synthesis of further PU nanocomposites. 


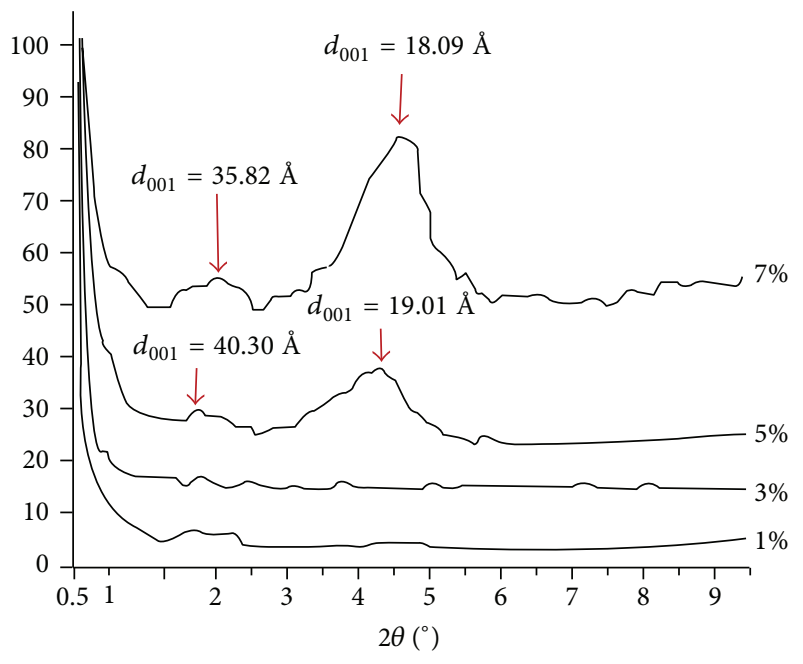

(a)

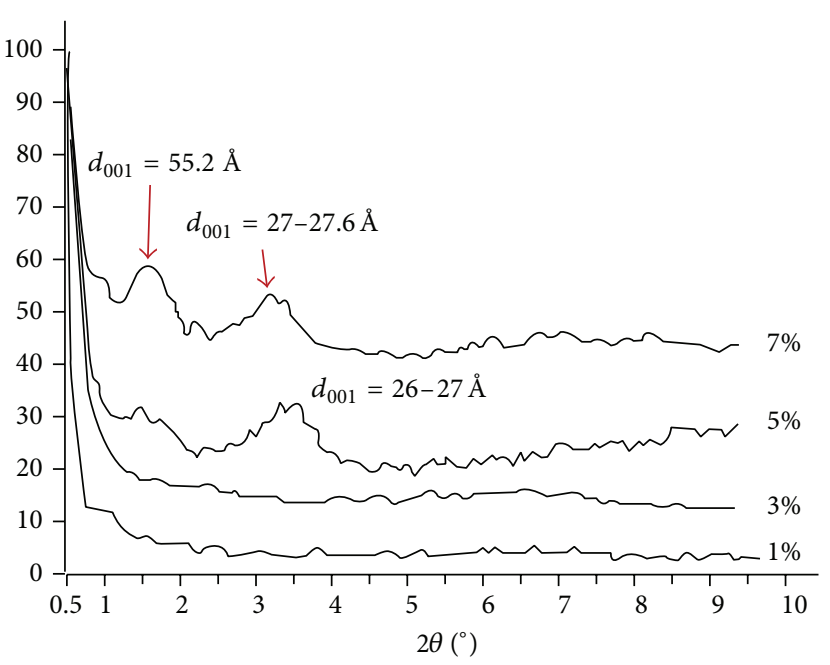

(b)

FIgURE 4: XRD patterns of the PU/organoclay nanocomposites reinforced by (a) MMT modified by PEO (PEO/MMT = 0.5); (b) MMT modified by PVA $(\mathrm{PVA} / \mathrm{MMT}=1)$.

\subsection{PU/Organoclay Nanocomposites}

3.3.1. WAXD Analysis. The PEO-MMT (PEO/MMT $=0.3)$ and PVA-MMT $(\mathrm{PVA} / \mathrm{MMT}=1)$ organoclays were used as reinforced phases in the PU nanocomposites. The XRD patterns of PU/PEO-MMT and PU/PVA-MMT nanocomposites are shown in Figure 4 . In both diagrams, the absence of detectable XRD peaks $\left(2 \theta=2-10^{\circ}\right)$ in 1 and $3 \%$ filler content PU nanocomposites might indicate the exfoliation and dispersion of the montmorillonite layers in the PU matrix.

Broad diffraction peaks are observed at $2 \theta=4.6^{\circ}\left(d_{001}=\right.$ $19 \AA)$ and $3.3^{\circ}\left(d_{001}=27 \AA\right)$ for PU/PEO-MMT and PU/PVAMMT, respectively, at organoclay loading rate of $5 \%$ and $7 \%$. A second peak showing another population of $\mathrm{d}$ spacing was observed at about 35-40 $\AA$ (PU/PEO-MMT) or $55 \AA$ (PU/PVA-MMT). This result shows that in the case of $5 \% \mathrm{wt}$ and $7 \% \mathrm{wt}$ loading, the silicate layers in the PU nanocomposites still mostly remain in the modified structure corresponding to the clear $d_{001}$ peak at $19 \AA$ for PEO-MMT and at $27 \AA$ for PVA-MMT. In other hand, another broaden peak found at a lower $2 \theta$ angle (35-40 $\AA$ ) in PU/PEO-MMT sample and at $55 \AA$ in PU/PVA-MMT sample, was referred to a part of PU chain which might intercalate and expand partially the organoclay gallery.

3.3.2. TEM Analysis. The direct measure of the nanometerscale dispersion of the layered silicates in the PU matrix can be observed in the cross-section TEM micrographs of the PU nanocomposites. Figure 5 shows the TEM images of $3 \%$ and 7\% PEO and PVA modified MMT in PU samples.

The lattice fringe of the organoclay silicate layers (darker line) were observed in the PU matrix. At 3\% filler loading rate, in the case of PEO-modified MMT (Figure 5(a)), the clay layers are nearly separated from each other with some interlayer spacing larger than the one of raw MMT distance (close to $1.5 \mathrm{~nm}$ ). While the organoclay modified by PVA are arranged in 4-7 layers stacking in disorder. This is evidence that these organoclays are intercalated in PU. Only, very few layers, completely separated from others, are exfoliated.

At a higher organoclay content, for $7 \%$ of PEO and PVA modified clay (Figures 5(b) and 5(d)), the layered filler is arranged in intercalated periodic stacking with 1.5$2 \mathrm{~nm}$ almost regular $\mathrm{d}$-spacing. In this case, the effective entry of PU molecules in between the organically modified interlamellar spacing could not be achieved to cause an exfoliation of the silicate layers in PU.

3.3.3. FTIR Analysis. As reported in the literature $[8,9$, $17,18]$, one important reason for the good dispersion of modified clay in the materials (at $3 \mathrm{wt} \%$ filler) is the strong interaction between the PU chains and clays modified by hydroxyl agents. This interaction may be due to either the formation of hydrogen bond between the - $\mathrm{OH}$ groups of intercalated polyols agent (PVA) and carbonyl groups of PU or to the bound amino group of urethane linkage attach to the layer surface $\mathrm{O}$ atoms through a $\mathrm{H}$ bond (in the case of PEO modified clay). The modified montmorillonites were extracted by soxhlet in $48 \mathrm{~h}$ using DMF as an extraction solvent from the PU/modified MMT nanocomposites and they were compared to pristine modified clay for their FTIR signals (Figure 7), in order to study the interaction of PU matrix with the modified clay. If there is a strong hydrogen interaction between PU chains and modified clay surface, the residual PU chains must be detected in clay surface after extraction.

The IR spectra of the extracted filler (Figure 7) illustrated the evidence of an interaction between clay and polymer chains in the form of physically adsorbed PU chains. The band at $2980-2864 \mathrm{~cm}^{-1}$ in the extracted filler and the raw modified MMTs are attributed to the asymmetric and symmetric $\mathrm{C}-\mathrm{H}$ stretching vibrations. The band of $\mathrm{C}=\mathrm{O}$ stretching vibrations forms a doublet in the extracted filler spectra, 


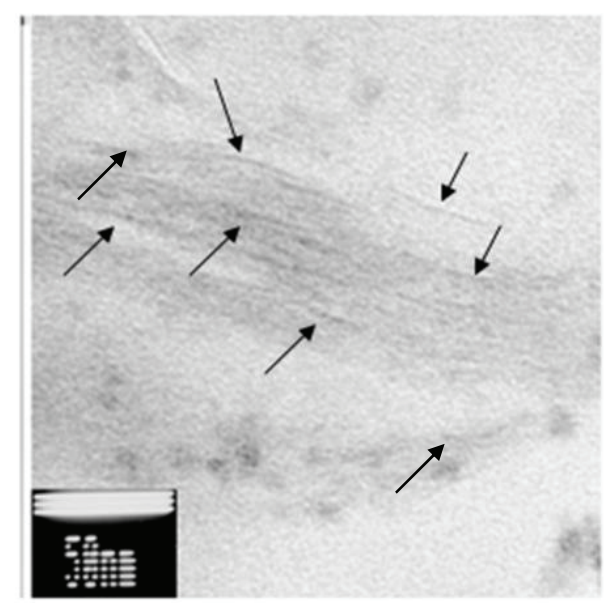

(a)

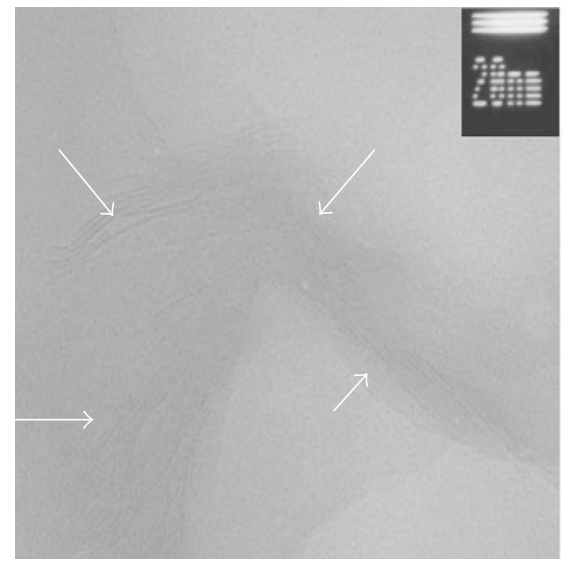

(c)

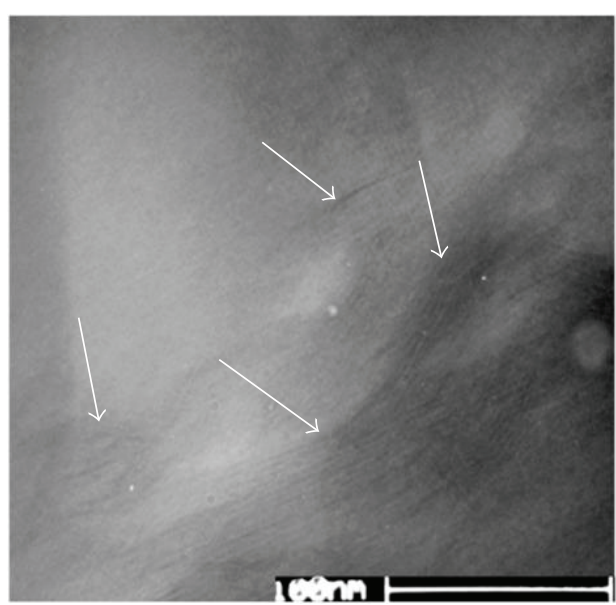

(b)

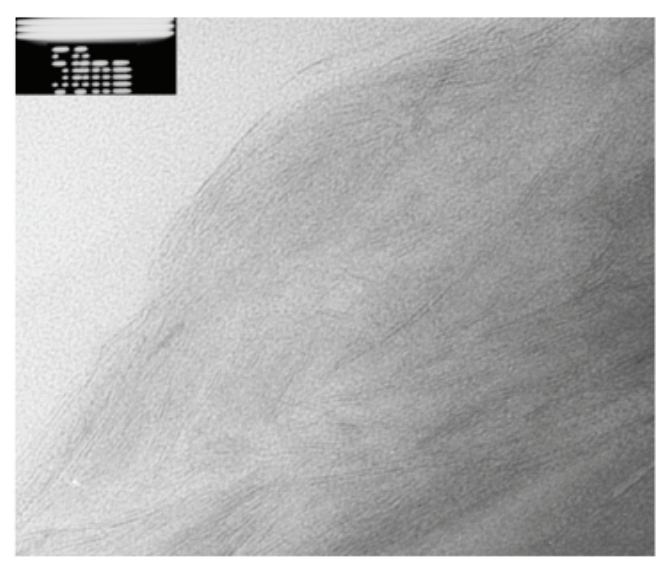

(d)

FIGURE 5: TEM images of PU nanocomposites prepared from organoclay modified by (a) PEO-MMT at 3\%wt; (b) PEO-MMT at 7\%wt; (c) PVA-MMT at 3\%wt, and (d) PVA-MMT at 7\%wt.

consisting with the free $\mathrm{C}=\mathrm{O}$ band at $1730 \mathrm{~cm}^{-1}$, and the carbonyls group vibration interacting with hydrogen bonds which are shifted to $1720 \mathrm{~cm}^{-1}$ especially in the case of PVA modified clay (shown in Figure 7). This implies that the hydrogen interaction has occurred between the urethane $\mathrm{C}=\mathrm{O}$ group and $\mathrm{OH}$ group of PVA whose chains are attached to surface layer through a $\mathrm{H}$ bond (Figure 6). This explanation is based on the study of Pattanayak and Jana [23] who has used the FT-IR analysis to prove the hydrogen interaction between $\mathrm{C}=\mathrm{O}$ of urethane linkage and alkylammonium $\mathrm{OH}$ group and reported that if the organically modified montmorillonite acted as chain extender; it was observed that the reactive clay was completely exfoliated in polyurethane matrix. The explanation from these authors [18, 23, 24, 28] might be adequate to our situation using PVA as the modifier, in which some polymer chain ends with - NCO groups diffused to the vicinity of the clay galleries during nanocomposites preparation and might react with $-\mathrm{OH}$ group of PVA modifier to produce urethane linkage, $-\mathrm{CO}-\mathrm{NH}-$. The urethane linkages, in turn, formed hydrogen bonds with the second $-\mathrm{OH}$ group residing on the other PVA chain inside the clay galleries, as illustrated in Figure 6(b). Meanwhile, this hydrogen interaction cannot happen in case of PEO modified clay, but only the hydrogen interaction between the ethoxy group of PEO and amide group of urethane linkage may occur (Figure 6(a)). That is why the peak of hydrogen liaison $\mathrm{C}=\mathrm{O}$ stretching at $1720 \mathrm{~cm}^{-1}$ is not observed in this case (Figure 7).

This phenomenon might bring out that these remarkable hydrogen interactions between clay surface and polymer chain would explain partly the better dispersion of clay in matrix. This result is similar to those obtained by Rehab and Salahuddin [25] or Pattanayak and Jana [18].

3.3.4. Thermal Behavior Analysis. The thermal properties of pure PU, PU/PEO-MMT, and PU/PVA-MMT nanocomposites were studied by DSC in the range $-100^{\circ} \mathrm{C}$ to $250^{\circ} \mathrm{C}$ (Figure 8). The DSC curves show that the glass transition temperature of $\mathrm{PU} /$ organoclay nanocomposites lie between $-28^{\circ} \mathrm{C}$ and $-29^{\circ} \mathrm{C}$, nearly identical to that of pure $\mathrm{PU}$ at $-28^{\circ} \mathrm{C}$ [28-30]. The effect of small amounts of dispersed modified MMT in the free volume of PU has no significant influence on 


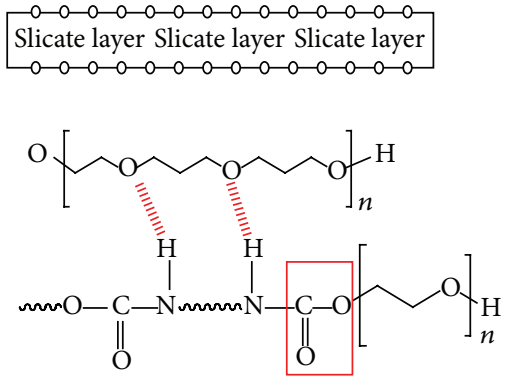

(a)

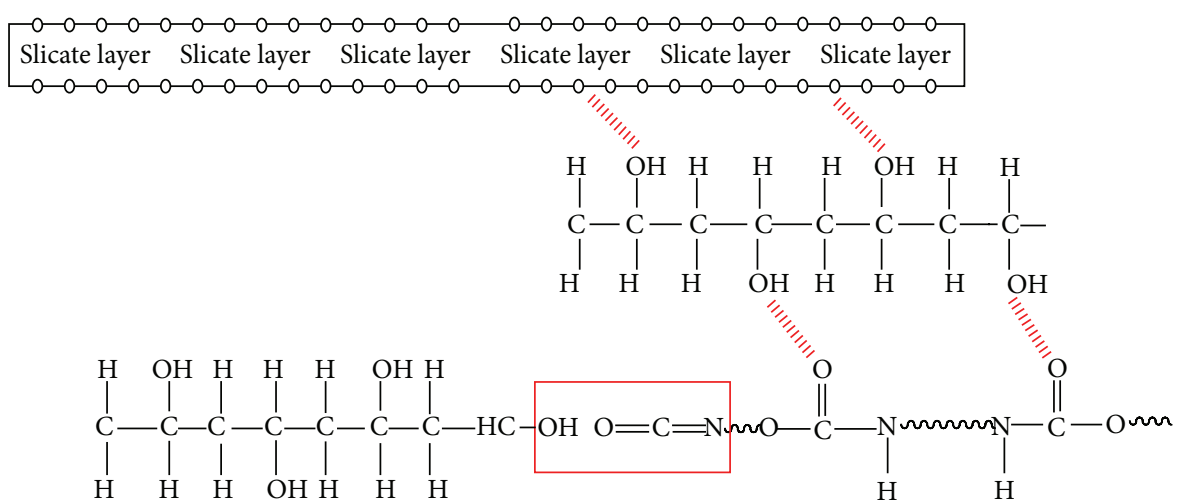

(b)

FIGURE 6: Schematic representation of the hydrogen bonding by clay-tethered polyurethane chain.

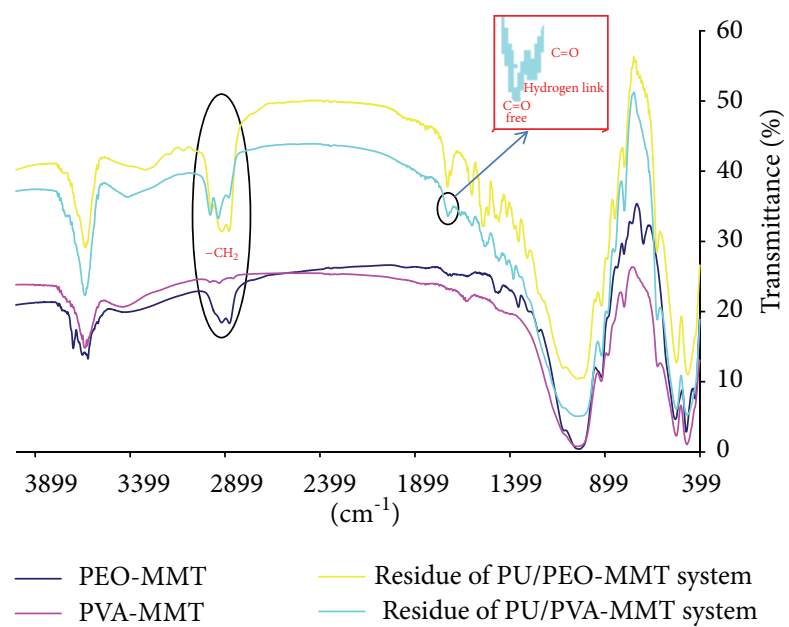

FIGURE 7: Infrared spectra of the organoclay (PEO-MMT and PVA$\mathrm{MMT}$ ) and the residues of PU/organoclay nanocomposites.

the glass transition temperature of PU. In the zone of product melting point, the endothermic peak area of PU/PEO-MMT and PU/PVA-MMT is considered to be fairly larger than that of pure PU (figure not shown in the case of PU/PVA$\mathrm{MMT}$ ), which means that organoclay did give some effect on the morphology of thermoplastic PU nanocomposite. This result is adequate to that of Chen et al. [21]. At the content of $3 \mathrm{wt} \%$ PEO, PVA-MMT and $5 \mathrm{wt} \%$ PVA-MMT loading (Table 1) in the nanocomposite, the increase of the melting temperature of material and the melting peak area promote the ordering in hard segments and the demixing of soft and hard segment $[21,31]$. At the higher contents of clay in the nanocomposites, the melting temperature of material seems to be decreased. This phenomenon is due to the aggregation of clay resulting from the mobility enhancement of the hard segment in material.

The thermal stability of PU, PU/PEO-MMT, and PU/PVA-MMT nanocomposite films was also investigated by TGA (data in Table 2).The degradation process passed

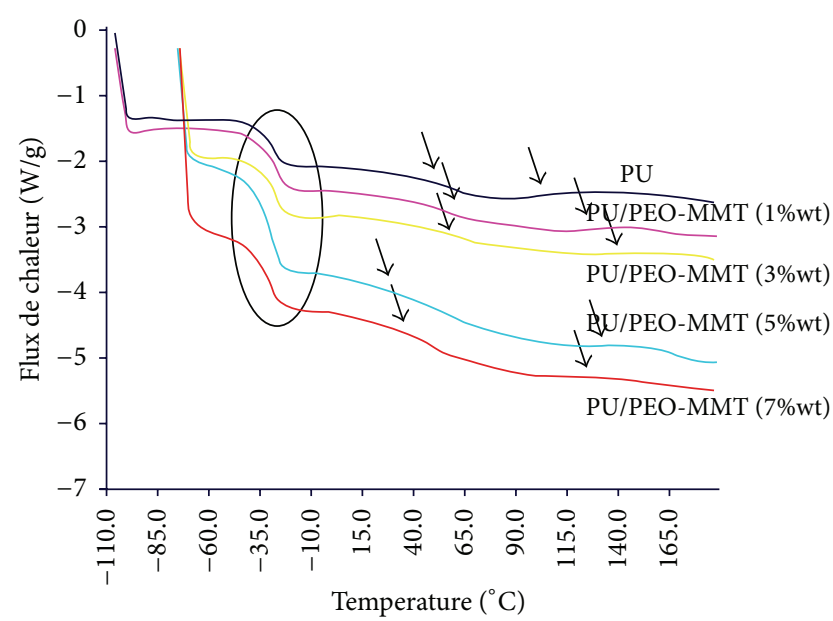

$\begin{array}{ll}\text { PU } & \text { PU/PEO-MMT (5\%wt) } \\ \text { PU/PEO-MMT (1\%wt) } & \text { PU/PEO-MMT (7\%wt) } \\ \text { PU/PEO-MMT (3\%wt) } & \end{array}$

FIgURE 8: The DSC curves of PU/PEO-MMT nanocomposites as a function of PEO-MMT filler loading content.

TABLE 1: DSC analysis data for the PU/PEO-MMT nanocomposites at different contents of PEO-MMT.

\begin{tabular}{lccc}
\hline Sample & $\begin{array}{c}\text { PEO-MMT loading } \\
\text { (\% massique) }\end{array}$ & $\begin{array}{c}T_{g} \text { of soft } \\
\text { segment }\left({ }^{\circ} \mathrm{C}\right)\end{array}$ & $\begin{array}{c}T_{m} \text { of } \\
\text { material }\left({ }^{\circ} \mathrm{C}\right)\end{array}$ \\
\hline \multirow{3}{*}{ PU/PEO-MMT } & 0 & -28 & 77 \\
& 3 & -28 & 76 \\
& 5 & -29 & 81 \\
& 7 & -28 & 73 \\
PU/PVA-MMT & 1 & -28 & 72 \\
\hline & 3 & -28 & 77 \\
& 5 & -26 & 80 \\
& 7 & -27 & 82 \\
\hline
\end{tabular}


TABLE 2: Thermal analysis data of polyurethane nanocomposites for different loading contents of PEO-MMT.

\begin{tabular}{|c|c|c|c|c|c|c|}
\hline \multirow[b]{2}{*}{ System } & \multirow{2}{*}{$\begin{array}{l}\text { PEO-MMT load } \\
\text { (\% of mass) }\end{array}$} & \multirow[b]{2}{*}{$T_{\mathrm{ID}}{ }^{*}\left({ }^{\circ} \mathrm{C}\right)$} & \multirow[b]{2}{*}{$T_{\operatorname{maxl}}{ }^{* *}\left({ }^{\circ} \mathrm{C}\right)$} & \multirow[b]{2}{*}{$T_{\max 2}{ }^{* *}\left({ }^{\circ} \mathrm{C}\right)$} & \multicolumn{2}{|c|}{ Weight loss (wt\%) } \\
\hline & & & & & $\begin{array}{c}190-320 \\
\left({ }^{\circ} \mathrm{C}\right)\end{array}$ & $\begin{array}{c}320-570 \\
\left({ }^{\circ} \mathrm{C}\right)\end{array}$ \\
\hline $\mathrm{PU}$ & 0 & 196 & 314 & 570 & $\sim 78$ & $\sim 21.4$ \\
\hline \multirow{4}{*}{ PU/PEO-MMT } & 1 & 206 & 329 & 565 & $\sim 83$ & $\sim 16.1$ \\
\hline & 3 & 230 & 324 & 568 & $\sim 80.1$ & $\sim 17.2$ \\
\hline & 5 & 251 & 332 & 565 & $\sim 77$ & $\sim 18.1$ \\
\hline & 7 & 255 & 333 & 564 & $\sim 76$ & $\sim 17.6$ \\
\hline \multirow{4}{*}{ PU/PVA-MMT } & 1 & 210 & 320 & 568 & $\sim 79$ & $\sim 20.7$ \\
\hline & 3 & 235 & 327 & 571 & $\sim 83$ & 14.8 \\
\hline & 5 & 254 & 335 & 566 & $\sim 77$ & $\sim 18.3$ \\
\hline & 7 & 261 & 337 & 565 & $\sim 75$ & $\sim 19.5$ \\
\hline
\end{tabular}

${ }^{*} T_{\text {ID }}$ : initial decomposition temperature.

${ }^{* *} T_{\max 1}$ and $T_{\max 2}$ : maximal decomposition temperature at the first and second stage, respectively.

through three stages as usually observed in thermoplastic PU [32]. In the first and second stage (from $190^{\circ} \mathrm{C}$ to $320^{\circ} \mathrm{C}$ ), the urethane bonds decompose to form alcohols and isocyanates. Complete volatilisation of resulting chain fragments is hindered by dimerisation of isocyanates to carbodiimide, which react with the alcohol groups to give relatively stable substituted urea (second step) that decompose in the third stage $\left(310^{\circ} \mathrm{C}-570^{\circ} \mathrm{C}\right)$. Trimerisation of isocyanates may also occur under certain conditions to yield thermally stable isocyanurate rings. The final step is the high temperature degradation of these stabilised structures to yield volatile products and a small amount of carbonaceous char [32,33].

The temperatures at $10 \%$ weight loss of both series of nanocomposite films are slightly higher than that of pure PU. It shows that the thermal degradation temperature of the nanocomposites is enhanced compared to that of neat PU. The introduction of inorganic components into organic materials can enhance their thermal resistance, as the dispersed silicate layers hinder the permeability of volatile degradation products out of the material $[8,21,29,33]$. The increase in thermal stability could also be attributed to the high thermal stability of clay and the interaction between the clay particles and the polymer matrix. Similar trends have been reported in other papers [26, 29, 32]. Moreover, thermal stability increases with an increase in clay loading content.

3.3.5. Dynamic Mechanical Analysis. DMA was used at room temperature to examine the viscoelastic response of the nanocomposite material to cyclic deformation, namely, elastic modulus (including storage modulus $\mathrm{E}^{\prime}$ and loss modulus $\left.\mathrm{E}^{\prime \prime}\right)$ and loss factors ( $\tan \delta$ ) for PU/PVA-MMT and PU/PEOMMT-nanocomposites. The enhancement of storage modulus is directly attributed to the reinforcement provided by the dispersed silicate layers $[7,8,11]$ and storage modulus can also be affected by the interfacial interaction between silicate layers and polyurethane matrix. Therefore, storage modulus increased with increasing the clay loading content and their organoclay dispersion.
TABLE 3: Storage modulus of PU, PU/PEO-MMT, and PU/PVAMMT nanocomposites.

\begin{tabular}{lccc}
\hline Sample & $\begin{array}{c}\text { Loading content } \\
(\text { Weight \%) }\end{array}$ & $\begin{array}{c}\text { Storage modulus } \\
\left(\mathrm{E}^{\prime}, \mathrm{MPa}\right)\end{array}$ & $\tan \delta * 10$ \\
\hline $\mathrm{PU}$ & 0 & 3.12 & 1.39 \\
\hline \multirow{2}{*}{ PU/PEO-MMT } & 1 & 3.99 & 1.25 \\
& 3 & 4.28 & 1.10 \\
& 7 & 4.49 & 1.06 \\
PU/PVA-MMT & 1 & 4.21 & 1.16 \\
& 3 & 4.10 & 1.22 \\
& 5 & 4.85 & 1.14 \\
& 7 & 5.02 & 1.09 \\
& & 4.88 & 1.12 \\
\hline
\end{tabular}

Both systems showed a significant increase in the storage modulus based on the amount of MMT (Table 3 ). In the case of PU/PVA-MMT, this increase is higher than that for the PU/PEO-MMT. As mentioned above, this might be due to better dispersion of clay in the PU matrix resulting in the strong interactions between polyurethane matrix and layered silicates as shown in IR results $[18,21,22]$. Numerous studies in the literature reported the same result $[1,8]$. The loss factors $(\tan \delta)$ of the two PU nanocomposite systems decrease compared to PU, indicating that the addition of clay diminish the polymer chains mobility as previously reported in the literature [8].

3.3.6. Water Absorption Measurement. Properties of many materials change with the difference in water absorption. The present application of PU is limited because its excellent properties can be greatly affected by water absorption. For example, most of the mechanical properties decrease greatly with the increase of relative humidity.

In the presence of clay, the water absorption ratios of PU nanocomposites were nearly lower than that of pure PU (Table 4). This can be explained by a mechanism of water 
TABLE 4: Water absorption of PU/PEO-MMT and PU/PVA-MMT system.

\begin{tabular}{|c|c|c|c|c|c|}
\hline Materials & \multicolumn{5}{|c|}{ Absorption uptake of water (wt\%) after 122 hours } \\
\hline $\begin{array}{l}\text { Modified clay } \\
\text { content (wt } \%)\end{array}$ & 0 & 1 & 3 & 5 & 7 \\
\hline PU/PEO-MMT & \multirow{2}{*}{2.16} & 1.82 & 1.91 & 2.09 & 2.18 \\
\hline PU/PVA-MMT & & 1.68 & 1.71 & 1.77 & 2.10 \\
\hline
\end{tabular}

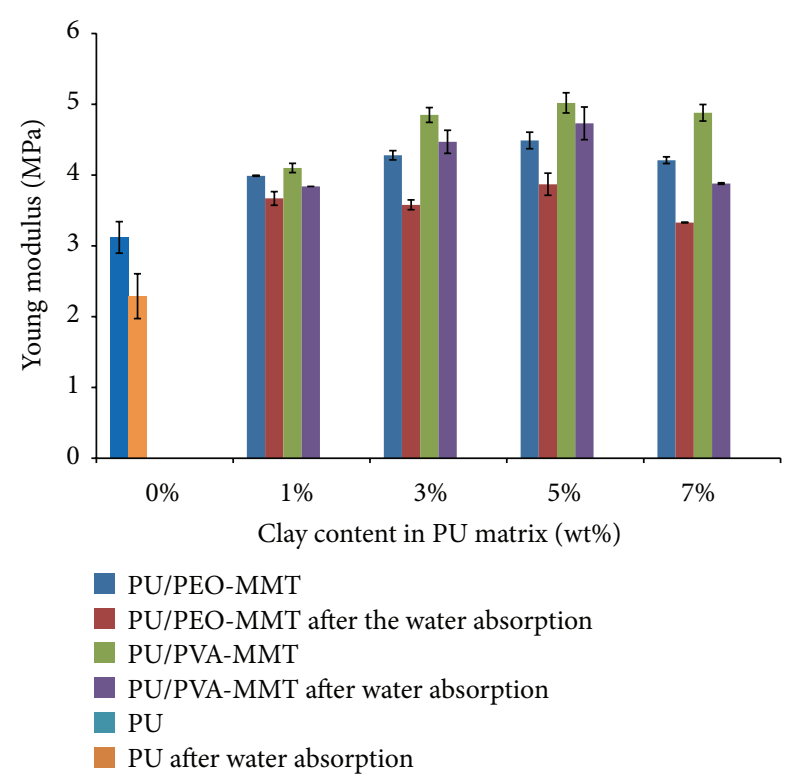

FIgUre 9: Young's modulus of PU, PU/PEO-MMT, and PU/PVAMMT nanocomposites before and after water absorption.

absorption controlled by two competing factors: the water absorption by the modified clay inside the nanocomposite (the absorption content of the nanocomposites will increase with the organoclay content) and the mean free path of water molecules to pass through the network of organoclay/PU increasing with the dispersion of the modified MMT in the PU matrix at a nanometer scale [21]. These two competitive effects result in the lowest water absorption for $1 \%$ wt of organoclay in PU (Table 4). While the load content of organoclay became more than $1 \%$, the clay-water absorption became dominant leading to slightly higher water absorption. In the case of PVA modified clay, the content of water absorbed into material is fewer than that of PEO modified clay case. This result is due to the better dispersion of organoclay in polymer matrix.

The Young's modulus of nanocomposite material is also measured after the water absorption experiment. By seeing in the histogram of Figures 9 and 10, both of the two cases, the loss of Young's modulus is proportional to the content of absorbed water. And at lower content, the effect of organoclay on the loss of modulus value seems to be not important. In addition, with the presence of PVA modified clay, the loss of modulus in material is lower than that using the PEO modified clay at any loading. This is due to the better dispersion of the filler modified by PVA.

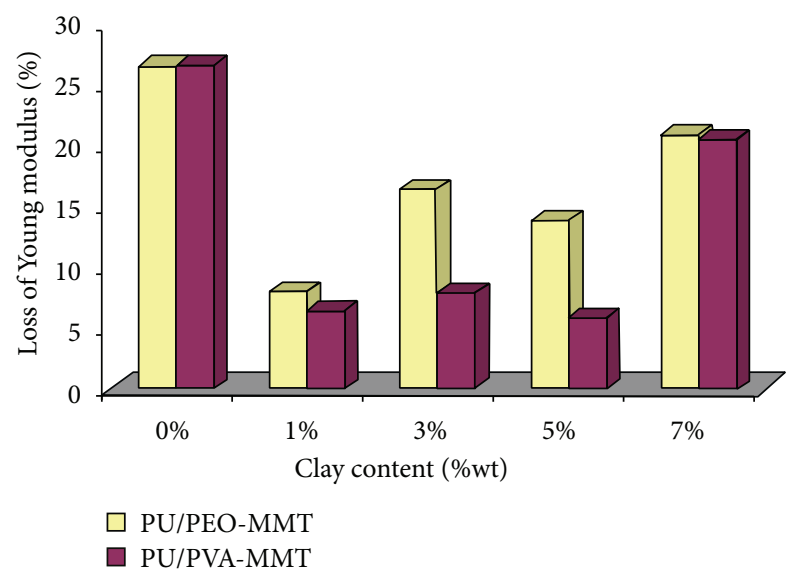

Figure 10: Young's modulus loss of PU, PU/PEO-MMT, and PU/PVA-MMT nanocomposites as a function of water absorption content.

\section{Conclusion}

The following conclusions are based on our observations involving the modification of clay and the elaboration of PU nanocomposite based on modified clay. The purified Vietnamese clay was successfully modified by two nonionic surfactants: PEO and PVA, and the result interlayer distance of MMT LD increases from 18 to $27 \AA$. Ester-TPU/clay nanocomposites were prepared by in situ polymerization using two types of modified clays. Morphological analysis showed that the $d$-spacing clearly increased with some disorder for low MMT content, whereas for higher content, the reinforced phase display the intercalated structures rearranged to a major extent. The introduction of inorganic components into organic materials can partially enhance their thermal resistance compared to that of neat PU. Young's modulus of material enhanced confirms the good dispersion of clays in PU matrix. But when the addition of organoclay reached more than $1 \%$, the water absorption function of clay became dominant, leading to slightly higher water absorption and resulted in the decrease of Young's modulus.

\section{Conflict of Interests}

Holding the copyright in this work, the authors of the paper would like to declare that this research does not involve any conflict of interests. The material sources for the study were all purchased from the commercial companies such as Clay Minerals Depository Hiep Phu Company and Sigma-Alrich Company Company.

\section{Acknowledgments}

The authors are grateful to the National University of Ho Chi Minh City, Vietnam, for the financial support and LCME lab of University of Savoie, France, for the support of material characterizations. 


\section{References}

[1] Z. Wang and T. J. Pinnavaia, "Nanolayer reinforcement of elastomeric polyurethane," Chemistry of Materials, vol. 10, no. 12, pp. 3769-3771, 1998.

[2] S. Solarski, S. Benali, M. Rochery et al., "Synthesis of a polyurethane/clay nanocomposite used as coating: interactions between the counterions of clay and the isocyanate and incidence on the nanocomposite structure," Journal of Applied Polymer Science, vol. 95, no. 2, pp. 238-244, 2005.

[3] G. W. Beall and S. J. Tsipursky, "Nanocomposites produced utilizing a novel ion-dipole clay surface modification," in Chemistry and Technology of Polymer Additives, S. Al-Malaika, A. Golovoy, and C. A. Wilkie, Eds., pp. 266-280, Blackwell Science, Oxford, UK, 1999.

[4] P. F. Luckham and S. Rossi, "Colloidal and rheological properties of bentonite suspensions," Advances in Colloid and Interface Science, vol. 82, no. 1, pp. 43-92, 1999.

[5] H. Sato, "Effects of the orientation of smectite particles and ionic strength on diffusion and activation enthalpies of $\mathrm{I}^{-}$and $\mathrm{Cs}^{+}$ ions in compacted smectite," Applied Clay Science, vol. 29, no. 3-4, pp. 267-281, 2005.

[6] A. Pacuła, E. Bielańska, A. Gaweł, K. Bahranowski, and E. M. Serwicka, "Textural effects in powdered montmorillonite induced by freeze-drying and ultrasound pretreatment," Applied Clay Science, vol. 32, no. 1-2, pp. 64-72, 2006.

[7] M. Alexandre and P. Dubois, "Polymer-layered silicate nanocomposites: preparation, properties and uses of a new class of materials," Materials Science and Engineering R, vol. 28, no. 1, pp. 1-63, 2000.

[8] J. Xiong, Z. Zheng, H. Jiang, S. Ye, and X. Wang, "Reinforcement of polyurethane composites with an organically modified montmorillonite," Composites A, vol. 38, no. 1, pp. 132-137, 2007.

[9] Q. M. Jia, M. Zheng, H. X. Chen, and R. J. Shen, "Synthesis and characterization of polyurethane/epoxy interpenetrating network nanocomposites with organoclays," Polymer Bulletin, vol. 54, no. 1-2, pp. 65-73, 2005.

[10] S. Sinha Ray and M. Okamoto, "Polymer/layered silicate nanocomposites: a review from preparation to processing," Progress in Polymer Science, vol. 28, no. 11, pp. 1539-1641, 2003.

[11] P. C. Lebaron, Z. Wang, and T. J. Pinnavaia, "Polymer-layered silicate nanocomposites: an overview," Applied Clay Science, vol. 15, no. 1-2, pp. 11-29, 1999.

[12] Z. Shen, G. P. Simon, and Y.-B. Cheng, "Saturation ratio of poly(ethylene oxide) to silicate in melt intercalated nanocomposites," European Polymer Journal, vol. 39, no. 9, pp. 1917-1924, 2003.

[13] Z. Shen, G. P. Simon, and Y.-B. Cheng, "Comparison of solution intercalation and melt intercalation of polymer-clay nanocomposites," Polymer, vol. 43, no. 15, pp. 4251-4260, 2002.

[14] R. A. Vaia, B. B. Sauer, O. K. Tse, and E. P. Giannelis, "Relaxations of confined chains in polymer nanocomposites: glass transition properties of poly(ethylene oxide) intercalated in montmorillonite," Journal of Polymer Science B, vol. 35, no. 1, pp. 59-67, 1997.

[15] P. Aranda and E. Ruiz-Hitzky, "Poly(ethylene oxide)-silicate intercalation materials," Chemistry of Materials, vol. 4, no. 6, pp. 1395-1403, 1992.
[16] K. E. Strawhecker and E. Manias, "Structure and properties of poly(vinyl alcohol)/ $\mathrm{Na}^{+}$montmorillonite nanocomposites," Chemistry of Materials, vol. 12, no. 10, pp. 2943-2949, 2000.

[17] L. Pizzatto, A. Lizot, R. Fiorio et al., "Synthesis and characterization of thermoplastic polyurethane/nanoclay composites," Materials Science and Engineering C, vol. 29, no. 2, pp. 474-478, 2009.

[18] A. Pattanayak and S. C. Jana, "Synthesis of thermoplastic polyurethane nanocomposites of reactive nanoclay by bulk polymerization methods," Polymer, vol. 46, no. 10, pp. 32753288, 2005.

[19] C. Zilg, R. Thomann, R. Mülhaupt, and J. Finter, "Polyurethane nanocomposites containing laminated anisotropic nanoparticles derived from organophilic layered silicates," Advanced Materials, vol. 11, no. 1, pp. 49-52, 1999.

[20] A. Cheng, S. Wu, D. Jiang, F. Wu, and J. Shen, "Study of elastomeric polyurethane nanocomposites prepared from grafted organic-montmorillonite," Colloid and Polymer Science, vol. 284, no. 9, pp. 1057-1061, 2005.

[21] T. K. Chen, Y. I. Tien, and K. H. Wei, "Synthesis and characterization of novel segmented polyurethane clay nanocomposite via poly(epsilon-caprolactone)/clay," Journal of Polymer Science A, vol. 37, pp. 2225-2233, 1999.

[22] Y. I. Tien and K. H. Wei, "High-tensile-property layered silicates/polyurethane nanocomposites by using reactive silicates as pseudo chain extenders," Macromolecules, vol. 34, no. 26, pp. 9045-9052, 2001.

[23] A. Pattanayak and S. C. Jana, "Thermoplastic polyurethane nanocomposites of reactive silicate clays: effects of soft segments on properties," Polymer, vol. 46, no. 14, pp. 5183-5193, 2005.

[24] A. Pattanayak and S. C. Jana, "High-strength and low-stiffness composites of nanoclay-filled thermoplastic polyurethanes," Polymer Engineering and Science, vol. 45, no. 11, pp. 1532-1539, 2005.

[25] A. Rehab and N. Salahuddin, "Nanocomposite materials based on polyurethane intercalated into montmorillonite clay," Materials Science and Engineering A, vol. 399, no. 1-2, pp. 368-376, 2005.

[26] C.-N. H. Thuc, A.-C. Grillet, L. Reinert, F. Ohashi, H. H. Thuc, and L. Duclaux, "Separation and purification of montmorillonite and polyethylene oxide modified montmorillonite from Vietnamese bentonites," Applied Clay Science, vol. 49, no. 3, pp. 229-238, 2010

[27] C. N. H. Thuc, Purification, intercalation/exfoliation of natural montmorillonite for elaboration of PU nanocomposite [Ph.D. thesis], University of Savoie, 2008.

[28] I. Clemitson, Castable Polyurethane Elastomers, CRC \& Taylor \& Francis, Boca Raton, Fla, USA, 2008.

[29] J.-H. Chang and Y. U. An, "Nanocomposites of polyurethane with various organoclays: thermomechanical properties, morphology, and gas permeability," Journal of Polymer Science B, vol. 40, no. 7, pp. 670-677, 2002.

[30] C. Jung, Synthesis of Thermoplastic Polyurethane and Polyurethane Nanocomposites under Chaotic Mixing Conditions, The Graduate Faculty of the University of Akron, 2005.

[31] C. H. Dan, M. H. Lee, Y. D. Kim, B. H. Min, and J. H. Kim, "Effect of clay modifiers on the morphology and physical properties of thermoplastic polyurethane/clay nanocomposites," Polymer, vol. 47, no. 19, pp. 6718-6730, 2006. 
[32] M. Berta, C. Lindsay, G. Pans, and G. Camino, "Effect of chemical structure on combustion and thermal behaviour of polyurethane elastomer layered silicate nanocomposites," Polymer Degradation and Stability, vol. 91, no. 5, pp. 1179-1191, 2006.

[33] W. J. Choi, S. H. Kim, Y. Jin Kim, and S. C. Kim, "Synthesis of chain-extended organifier and properties of polyurethane/clay nanocomposites," Polymer, vol. 45, no. 17, pp. 6045-6057, 2004. 

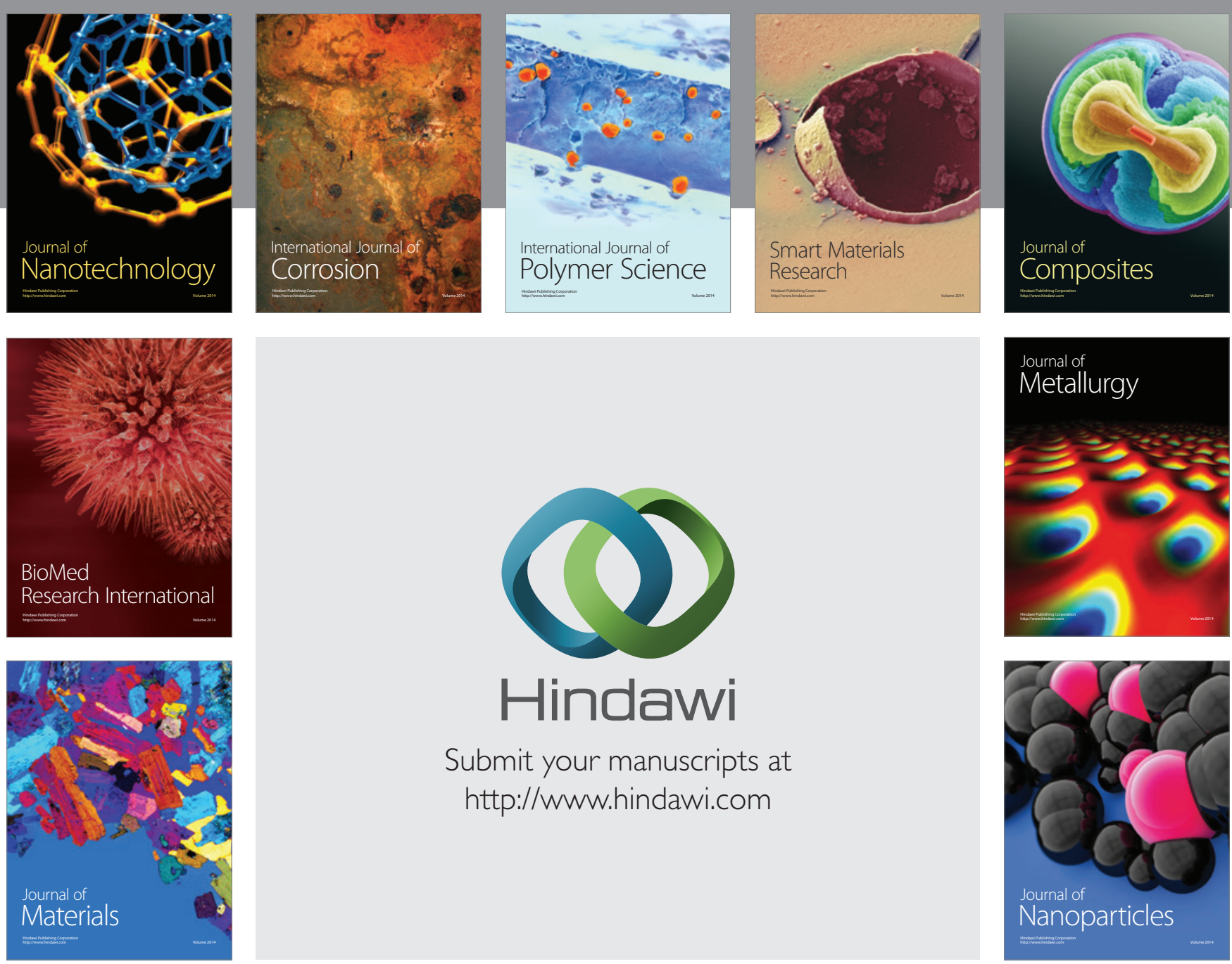

Submit your manuscripts at http://www.hindawi.com
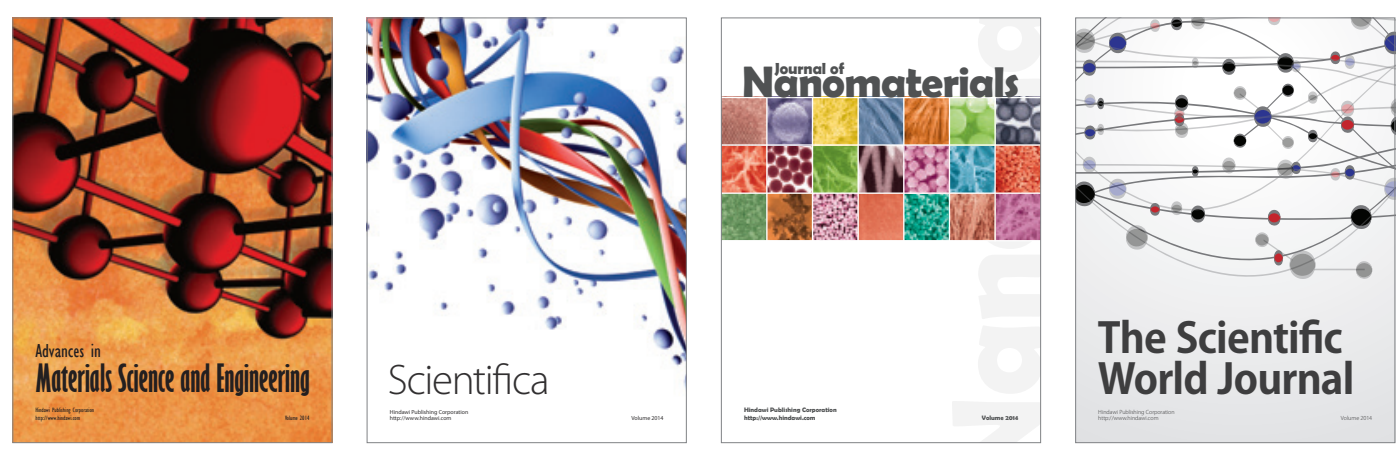

\section{The Scientific World Journal}
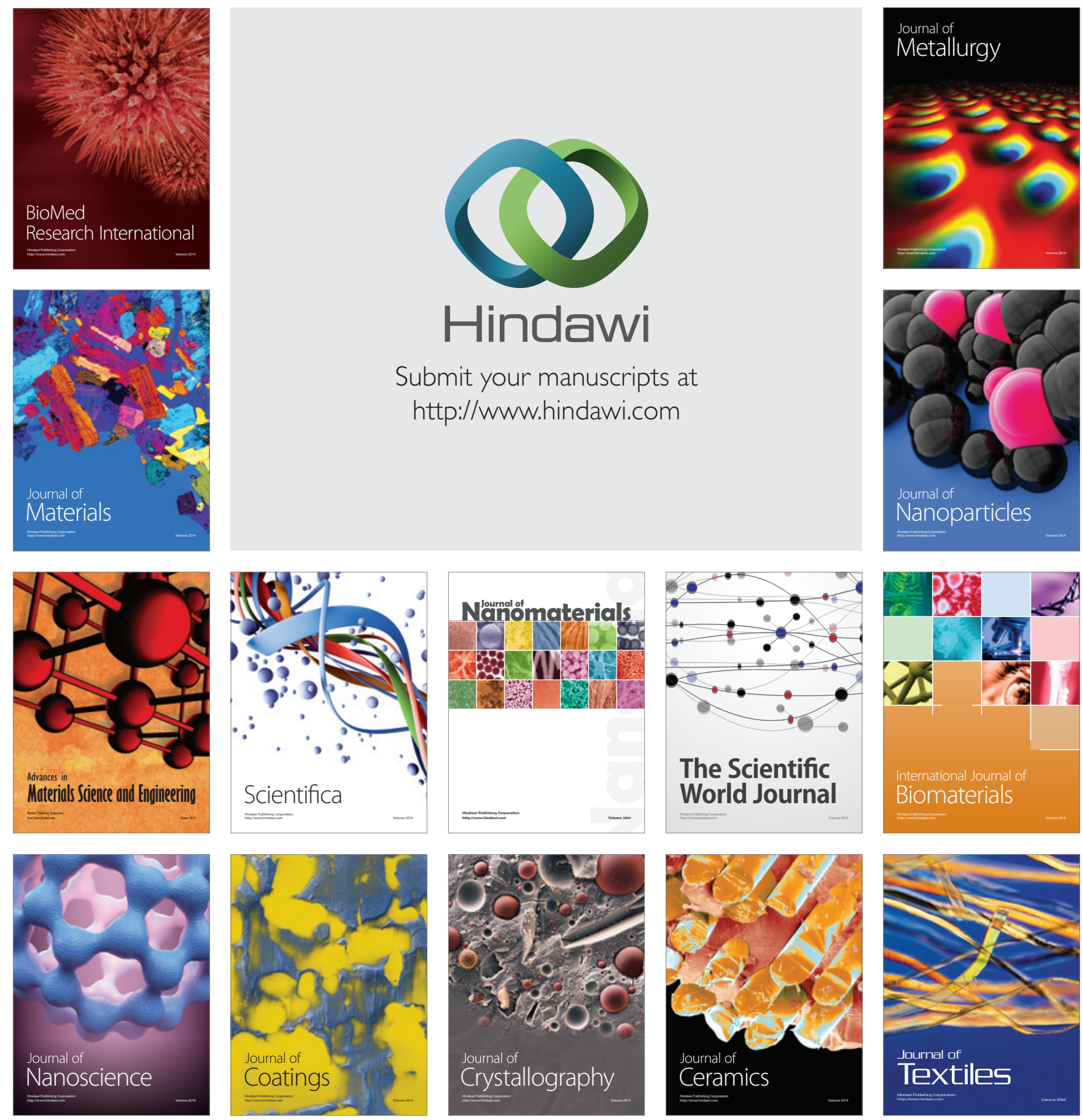